\title{
Unknown GBS Risk Status
}

National Cancer Institute

\section{Source}

National Cancer Institute. Unknown GBS Risk Status. NCI Thesaurus. Code C111949.

Rectal/vag inal culture not taken within 5 weeks prior to birth, or urine GBS culture not taken. 